\title{
The Effect of Nutrient-Dense, Portion-Controlled Functional Foods in Overweight Men with Reduced Libidos: A Prospective, Pilot Study
}

\author{
Kate Bauer ${ }^{1}$, Greg J Sommer ${ }^{2}$, Marra S Francis ${ }^{3}$, Vasuki Wijendran ${ }^{1}$, Charles Marsland ${ }^{4}$ and Stacey J Bell*5 \\ ${ }^{1}$ Senior Clinical Research Associate, Nutrient, USA
}

${ }^{2}$ Founder and Chief Scientific Officer, Sandstone Diagnostics, USA

${ }^{3}$ Executive Medical Director, Everlywell, Inc, USA

${ }^{4}$ Founder and Vice Chairman, Nutrient, USA

${ }^{5}$ Chief Science Officer, Nutrient, USA

Submission: November 25, 2019; Published: December 09, 2019

*Corresponding author: Stacey J Bell, D.Sc., RDN, Chief Science Officer, Nutrient, 110 Woodland Street Reno, NV 89523, USA

Abstract

Background and Objectives: Aging and weight gain often lead to reduced male libido, testosterone levels, and reproductive health functions. Younger men, who have reduced libido due to no under-lying medical or environment cause, are often prescribed medications. Nonpharmacological treatment usually includes weight loss.

Materials and Methods: The aim of this prospective, single-arm, four-month study was to evaluate the effect of a nutrient-dense dietary plan using portion-controlled, pre-packaged functional foods on body weight, sexual performance, free testosterone, semen volume, and sperm concentration in overweight men aged 20-50 years of age.

Results: Eleven individuals began the study, and six completed it. Participants consumed either four or five nutrient-rich functional foods daily and ate mostly healthy foods otherwise. Baseline mean weight was $107 \pm 21 \mathrm{~kg}$, decreasing to $97 \pm 24$ by week 8 , but was $104 \pm 19$ at week 16 . Mean waist circumference decreased at each measurement: $112 \pm 13 \mathrm{~cm}$ at baseline, $110 \pm 12 \mathrm{~cm}$ at week 8 , and $108 \pm 11 \mathrm{~cm}$ at week 16 . Everyone, except one subject who had no change, experienced a decrease in waist circumference of $2-8 \mathrm{~cm}$. All participants for whom data were available experienced increases in salivary testosterone concentrations between 18 and $87 \mathrm{pg} / \mathrm{mL}$; by week 4, all had normal readings. At baseline and throughout the study, all but one person had normal semen volumes (i.e., at least $1.5 \mathrm{~mL}$ ). Sperm concentrations were Optimal at baseline for three participants (i.e., $\geq 55 \mathrm{M} / \mathrm{mL}$ ), and the remaining two had Moderate values (16-54 M/mL, inclusive). Sperm concentrations varied throughout the study with three men experiencing Moderate concentrations at week 4, and two had Low concentrations (i.e., $\leq 15 \mathrm{M} / \mathrm{mL}$ ). At week 8, all five subjects had Moderate sperm concentrations. By week 12, four had Moderate readings and one had an Optimal sperm concentration; at week 16, this improved to three with Moderate readings and two had Optimal concentrations. Total sperm counts (semen volume x sperm concentration [in millions]) varied among the participants. Between baseline and week 16, two participants had a lower total sperm count (decrease of $12 \%$ and 50\%), and three had increased counts (all had 40-45\% increases). Sexual health was assessed via a questionnaire (Sexual Health Inventory for Men [SHIM]). Upon entry into the study, all participants had suboptimal SHIM scores (i.e., $\leq 21$ ); at the end, three of the six normalized. For the general quality of life questions, scores tended to be better for each quality of life indicator, except gastrointestinal issues (GI), which worsened over time. The greatest improvements were seen in energy level, diet quality, and passion.

Conclusion: Overweight men with low libidos experienced benefits from consuming nutrient-dense functional foods for anthropometric measurements, quantitative measures of testosterone, semen volume, sperm concentration, and subjective measures of quality of life. These preliminary results are encouraging, and we are continuing to recruit more subjects to confirm these findings.

Keywords: Low libido; Weight loss; Functional foods; Nutrient-dense foods; Diabetes; Thyroid disorders; Iatrogenic factors; Subfertility; Overweight men; Testosterone; Semen volume; Sperm concentration

Abbreviations: BMI: Body Mass Index; GI: Gastrointestinal Issues; SHIM: Sexual Health Inventory for Men; CASA: Computer Aided Semen Analysis; CVD: Cardiovascular Disease 


\section{Introduction}

Low libido in men can occur at any age but is more common in older individuals who have lower testosterone levels [13]. However, in a systematic review including 40 studies, the percentage with low testosterone levels varied between $2 \%$ and $77 \%$ in men at all ages, indicating that age may not be the only factor [4]. The wide range of low testosterone concentrations may be attributed to using different measurements of testosterone (i.e., free, total, and bioavailable testosterone). Regardless of the prevalence today, mean testosterone concentrations are falling faster at all age groups compared to previous predictions [3]. By 2025 as many as 6.5 million American men aged 30-79 years will have symptomatic androgen deficiency related to low testosterone levels [1]. This represents an increase of 38\% since 2000 .

Men's reproductive health is experiencing a similar decline, with recent reports demonstrating that the average sperm count in Western men has dropped by more than $50 \%$ between the early 1970 s and 2011 [5]. Based on this systematic review and meta-analysis, sperm concentration declined 52\% (-1.4\% per year) and total sperm count declined by $59 \%$ ( $-1.6 \%$ per year). This male fertility decline is likely at least partly linked to the overall decline in testosterone levels as both sperm production and testosterone are markers of testicular function. However, a major driver of decreased reproductive health relates to obesity [6-9].

Obesity and increased waist circumference contribute to male subfertility by increasing estrogen concentrations, producing excess inflammatory compounds that induce oxidative stress in the male reproductive organs, or some other mechanisms [68]. Regardless of the mechanism, sperm quality is affected by obesity [9]. Based on a retrospective study of more than 1,200 males, obesity was associated with significantly lower semen volume, total sperm count, and sperm concentration.

Testosterone levels decrease with aging, but this is not the only cause of reduced libido [2,3]. Low libido can also be caused by fatigue, stressors (e.g., work, finance), depression, low selfesteem and relationship problems, especially when the sexual partner is discordant regarding sexual activity [2]. In addition, reduced libido may be related to common conditions such as diabetes, thyroid disorders, iatrogenic factors from medications (e.g., beta blockers, SSRIs), and lifestyle factors such as excess alcohol intake [2].

For younger males, the issue of low libido is rarely broached by primary care doctors because the physicians have a fear of embarrassing the patient or are not confident about their ability to provide treatment [2]. In addition, younger men are much less likely to regularly see a physician than women and older men, and thus much less likely to broach libido concerns with a physician.

Pharmacological treatment of reduced libido usually starts with one of four approved drugs for erectile dysfunction known as PDE type-5 inhibitors, which increase penile blood flow [10]. Non-pharmacologic treatments include eating a better diet with fewer processed and high-fat foods, maintaining a healthy body weight, stopping smoking, increasing exercise, limiting drug and alcohol use, and sleeping 7-8 hours nightly [2]. These nonpharmacologic treatments are also recommended for men trying to conceive as they have been linked to increased sperm health and fertility [11].

Non-pharmacological treatments to improve libido are welcome, especially if related to promoting weight loss. Functional foods have been proposed as a solution [12]. These foods are foods enhanced with bioactive ingredients and have demonstrated health benefits. Functional foods usually have demonstrated beneficial physiological effects in the prevention, management, and/or treatment of chronic disease that is beyond basic nutrition. The functional foods can come from conventional foods or from the addition of a bioactive constituent in conventional food like vitamins and minerals. Novel nutrientdense functional foods have already been shown to produce a medically significant weight loss $[13,14]$.

The purpose of this study was to explore the effect of diet, one of the non-pharmacological treatments of reduced libido and fertility, on body weight, sexual performance, free testosterone, semen volume, and sperm concentration in overweight men aged 20-50 years of age. Overweight men with low libidos are provided novel nutrient-dense functional foods, which have already been shown to produce weight loss $[13,14]$. It is possible that a healthy, nutrient-rich diet using functional foods may improve both libido and sperm health by promoting weight loss.

\section{Methods}

\section{Subjects}

Overweight and obese males (body mass indexes [BMI] $25-45 \mathrm{~kg} / \mathrm{m} 2$ ), aged 20-50 years were recruited through Don't Cook Your Balls (https://www.dontcookyourballs.com/) and social media. Men with the following conditions were excluded: (1) a vasectomy, (2) been diagnosed as azoospermic, (3) having hypogonadism, or (4) on testosterone-replacement therapy. In addition, those living in New York, New Jersey, Maryland and Rhode Island could not participate because certain state laws prevented the testosterone testing kits from being sent to participants living in these states.

\section{Diet}

Subjects were provided five nutrient-dense, portioncontrolled functional foods daily over four months. Foods were provided at no charge and were easy-to-prepare; most required just the addition of hot water. Participants could choose from a variety of options such as shakes, cold and hot cereals, food bars, and hot meals (e.g., Pad Thai, noodles and cheese sauce) (http://nutrientfoods.com). Each functional food contained at 
least $25 \%$ of the Daily Value (DV) for every vitamin and mineral, except sodium and chloride. At least $25 \%$ Adequate Intakes (AI) for omega-3s were also included in each offering. Free nutrition coaching was provided to assist with questions about the dietary intervention and aid compliance.

\section{Sexual health inventory for men (SHIM) questionnaire}

SHIM is a widely used tool for diagnosing erectile dysfunction and determining its severity [15]. The questionnaire is widely used in research studies and in clinical practice to further enhance the decision making of physicians. The highest total score is 25 and 0 is entered if intercourse was not attempted. A score of less than or equal to 21 is considered to be low and may require a physician visit. The participants took the SHIM test at baseline, week 4, week 8, week 12, and week 16 .

\section{General quality of life questionnaire}

Each week, subjects answered nine questions about their quality of life. Each was rated using a scale of one to five, with 5 being the best and 1 being the worst. Questions probed: general feeling, fullness, mood, energy level, any gastrointestinal symptoms (GI), sleep quality, appearance, overall diet quality, and passion. The percentage change between the mean at baseline and week 16 was calculated.

\section{Testosterone}

Salivary free testosterone was determined using a home testing kit (https://www.everlywell.com/products/ testosterone-test/). Detailed instructions were included in the kit, along with a shipping envelope. Free testosterone is expressed in $\mathrm{pg} / \mathrm{mL}$ using these criteria: Low <49.0; Normal 49.0-185; and High >185.0. Serum free testosterone correlates well with salivary measurements $(\mathrm{r}=0.71 ; \mathrm{P}<0.001)$ [16]. Testosterone was measured at baseline, week 4, week 8 , week 12, and week 16 .

\section{Semen volume and sperm concentration}

Semen volume and sperm concentration were measured using a home testing kit (https://trakfertility.com/buy-traknow/trak-male-fertility-testing-system/). This is the only home sperm test designed to provide a way to measure semen volume and sperm concentration. It is the only FDA-cleared device that determines sperm count as: Low, Moderate, or Optimal for conception. The sample collection cups used to determine semen volume are FDA cleared to diagnose hypospermia (low sperm volume) [17].

Normal semen volume is considered at or above $1.5 \mathrm{~mL}$. For sperm concentration, the following criteria were used: $\geq 55 \mathrm{M} /$ $\mathrm{mL}$, Optimal; 16-54 M/mL inclusive, Moderate; and $\leq 15 \mathrm{M} /$ $\mathrm{mL}$, Low and may want to consult a physician. The home testing kit was previously validated against a computer aided semen analysis (CASA) at three home-simulated sites in fertility centers or urology offices [17]. Results were comparable between the home testing kit and CASA measurements. When measured quantitatively, a strong linear correlation existed between the home test kit and CASA measurements ( $r=0.99)$. The home test had an accuracy (versus the reference) of $93 \%$ for results categorized as Low; $82 \%$ for results categorized Moderate; and as $95 \%$ for those deemed Optimal.

Participants measured semen volume and sperm concentration at baseline, week 4, week 8, week 12 and week 16. The participants took a photograph of their semen volume and sperm concentration results, which was then verified and quantified by one of us (GS). From these direct measurements, total sperm counts (in millions) were calculated by semen volume x sperm concentration.

\section{Study design}

Once potential candidates were identified, extensive screening followed. Participants were reminded that during the four-month study they needed to:

a) Consume five nutrient-dense functional foods, which would constitute most of their diet

b) Conduct self-testing of free testosterone, semen volume, and sperm concentration at baseline and at weeks $4,8,12$, and 16

c) Complete and submit weekly data collection forms electronically

d) Stay in regular contact with their nutrition coach

Before the nutrient-dense functional foods were consumed, participants measured testosterone, semen volume, sperm concentration and completed the SHIM questionnaire. These activities were performed again at weeks 4, 8, 12, and 16 . After the nutrient-dense foods were introduced, each week, participants electronically submitted data about body weight, compliance with meals, general quality of life, exercise, and use of alcohol, smoking (tobacco and marijuana), and illicit drugs. Disease risk for chronic diseases (i.e., hypertension, type 2 diabetes, and cardiovascular disease [CVD]) was determined from body weight and waist circumference at baseline and the end of the study [18].

\section{Statistics}

All data are reported as mean \pm standard deviation (S.D.).

\section{Results}

Eleven men enrolled in the study with an average age of $36 \pm 6$ years (Table 1). One was classified as being Overweight and the remaining were Obese. Five withdrew early; one due to gastrointestinal issues related to the nutrient-dense functional foods and four did not submit the required data. One remained in the study, but did not conduct tests for testosterone, semen volume, or sperm concentration. The participants had other conditions beside a low libido: anxiety, depression, PTSD, ADHD, sleep apnea, hypothyroidism, and fatigue. Two reported using 


\section{Current Research in Diabetes \& Obesity Journal}

a medication for erectile dysfunction. The use of illicit drugs, smoking, and excessive alcohol use was non-existent at baseline and throughout the study.

Table 1: Baseline demographics.

\begin{tabular}{|c|c|}
\hline Attribute & Number or Mean \pm Standard Deviation \\
\hline Number of subjects & 11 \\
\hline Age (years) & $36 \pm 6$ \\
\hline Body Mass Index $\left(\mathrm{kg} / \mathrm{m}^{2}\right.$ ) & $34 \pm 4$ \\
\hline Classification of BMI & $\begin{array}{c}\text { 1=0verweight; 7=Obese (class 1); 1=0bese } \\
\text { (class 2); 2=Obese (class 3) }\end{array}$ \\
\hline $\begin{array}{c}\text { Waist Circumference } \\
(\mathrm{cm})\end{array}$ & $\begin{array}{c}115 \pm 11 \text { (high risk } \geq 102 \mathrm{~cm} \text { ) (9/11 were } \\
\text { high risk) }\end{array}$ \\
\hline Disease Risk* & 3=High; 6=Very High; 2=Extremely High \\
\hline
\end{tabular}

*Disease risk for type 2 diabetes, cardiovascular disease, and hypertension [18].

Of the six remaining participants, half consumed all five prescribed functional foods daily throughout the study, while the others consumed four. In addition, they ate relatively healthy foods such as fruits, vegetables, and low-fat protein. Consumption of ultra-processed foods (e.g., baked goods, chips, sugar-sweetened beverages) was minimal.

The group engaged in a variety of exercises: use of the elliptical, strength training, yoga, walking, jogging, CrossFit, martial arts, and using Insanity cardio videos. Three participants exercised around 220 minutes per week and the other three exercised 50-100 minutes weekly.

\section{Anthropometry}

For the first two months, the participants lost weight but afterwards there was weight regain (Table 2). Baseline mean weight was $107 \pm 21 \mathrm{~kg}$, decreasing to $97 \pm 24$ by week 8 , and increasing to $104 \pm 19$ at week 16 , indicating a $3 \%$ loss during the study. Mean body mass index (BMI) followed this pattern of decreasing early on and increasing towards the end. At the end of the study, one participant lost $13.6 \mathrm{~kg}$; three lost $2-3 \mathrm{~kg}$, one had no change, and one gained $6.4 \mathrm{~kg}$.

Table 2: Changes in anthropometry.

\begin{tabular}{|c|c|c|c|}
\hline & Weight (kg) & $\begin{array}{c}\text { Body Mass Index } \\
\left(\mathbf{k g} / \mathbf{m}^{\mathbf{2}} \mathbf{)}\right.\end{array}$ & $\begin{array}{c}\text { Waist Circumfer- } \\
\text { ence (cm) }\end{array}$ \\
\hline Baseline & $107 \pm 21$ & $33 \pm 5$ & $112 \pm 13$ \\
\hline Week 4 & $98 \pm 25$ & $31 \pm 7$ & $109 \pm 14$ \\
\hline Week 8 & $97 \pm 24$ & $31 \pm 7$ & $110 \pm 12$ \\
\hline Week 12 & $101 \pm 24$ & $31 \pm 5$ & $108 \pm 12$ \\
\hline Week 16 & $104 \pm 19$ & $33 \pm 4$ & $108 \pm 11$ \\
\hline
\end{tabular}

Mean waist circumference decreased at each measurement: $112 \pm 13 \mathrm{~cm}$ at baseline, $110 \pm 12 \mathrm{~cm}$ at week 8 , and $108 \pm 11 \mathrm{~cm}$ at week 16. Except one subject who had no change, everyone else experienced a decrease in waist circumference of $2-8 \mathrm{~cm}$.
Based on BMI and waist circumference, chronic disease risk reduced during the study (18). At baseline, three were at High risk, six were at Very High, and two were at Extremely High risk. After 16 weeks, of the six remaining participants, no one was at Extremely High risk, fewer were at Very High risk (four, down from six at the beginning of the study), and one was in both the High and Increased risk categories (data not shown).

\section{Testosterone}

Based on four men (two of six did not submit samples after baseline), all experienced increased salivary testosterone concentrations over the four months (Table 3). At baseline, two had low readings and two were normal. By week 4 and continuing to the end, all had normalized readings. Participants had increases between 18 and $87 \mathrm{pg} / \mathrm{mL}$. Weight loss was not correlated with testosterone levels.

Table 3: Changes in salivary free testosterone concentrations*.

\begin{tabular}{|c|c|c|c|c|c|}
\hline $\mathbf{p g} / \mathbf{m L}$ & Baseline & Week 4 & Week 8 & Week 12 & Week 16 \\
\hline Participant 1 & 38 & 78 & 85 & 54 & 56 \\
\hline Participant 2 & 87 & 160 & 64 & 84 & 174 \\
\hline Participant 3 & 47 & 60 & 169 & N/A & 102 \\
\hline Participant 4 & 73 & 73 & 109 & 75 & 133 \\
\hline
\end{tabular}

*Normal salivary testosterone: $49-185 \mathrm{pg} / \mathrm{mL}$

\section{Semen volume and sperm concentration}

Table 4: Changes in semen volume, sperm concentrations, and total sperm counts*.

\begin{tabular}{|c|c|c|c|c|c|}
\hline $\begin{array}{c}\text { Semen Volume } \\
\text { (mL) } \\
\text { Sperm Concen- } \\
\text { tration (M/mL) } \\
\text { Total Sperm } \\
\text { Count } \\
\text { (millions) }\end{array}$ & $\begin{array}{l}\text { Base- } \\
\text { line }\end{array}$ & Week 4 & Week 8 & $\begin{array}{c}\text { Week } \\
12\end{array}$ & $\begin{array}{c}\text { Week } \\
16\end{array}$ \\
\hline \multirow{3}{*}{ Participant 1} & 1.9 & 1.5 & 1.8 & 2.2 & 2.8 \\
\hline & 52 & 48 & 45 & 55 & 62 \\
\hline & 99 & 72 & 81 & 121 & 174 \\
\hline \multirow{3}{*}{ Participant 2} & 5 & 2.5 & 7 & 6 & 7 \\
\hline & 60 & 55 & 30 & 25 & 36 \\
\hline & 300 & 138 & 210 & 150 & 252 \\
\hline \multirow{3}{*}{ Participant 3} & 1.5 & 2.5 & 1.5 & 3 & 2.5 \\
\hline & 93 & 20 & 21 & 55 & 98 \\
\hline & 140 & 50 & 32 & 165 & 254 \\
\hline \multirow{3}{*}{ Participant 4} & 1 & 1 & 0.7 & 1 & 0.7 \\
\hline & 72 & 15 & 32 & 30 & 33 \\
\hline & 72 & 15 & 22 & 30 & 23 \\
\hline \multirow{3}{*}{ Participant 5} & 5 & 5 & 4.9 & 4 & 4 \\
\hline & 18 & 15 & 50 & 80 & 38 \\
\hline & 90 & 75 & 245 & 320 & 152 \\
\hline
\end{tabular}

* $\geq 55 \mathrm{M} / \mathrm{mL}$, Optimal; $16-54 \mathrm{M} / \mathrm{mL}$ inclusive, Moderate; and $\leq 15 \mathrm{M} /$ $\mathrm{mL}$, Low and may want to consult a physician 
Five participants submitted samples for semen volume and sperm concentrations and their results varied over the four months (Table 4). At baseline and throughout the study, all but one person had normal semen volumes (i.e., at least $1.5 \mathrm{~mL}$ ). At each 4-week measurement, all participants had normal semen volumes, except the one who was low at the onset, and continued to be low throughout the study. Two participants experienced decreases in volume $(-0.3$ and $-1.0 \mathrm{~mL})$ and the others had increased volumes of $0.9,1.0$, and $2.0 \mathrm{~mL}$.

At baseline, three participants had an Optimal sperm concentration (i.e., $\geq 55 \mathrm{M} / \mathrm{mL}$ ) and the remaining two subjects had Moderate concentrations (16-54 M/mL inclusive). Values varied throughout the study; at week 4 , three men had Moderate concentrations, and two had Low concentrations $(\leq 15 \mathrm{M} /$ $\mathrm{mL}$ ). At week 8, all five had Moderate readings. At week 12, four were Moderate and one had an Optimal reading $(\geq 55 \mathrm{M} / \mathrm{mL})$. At the end, two were Optimal and three were Moderate. Only one participant had an Optimal concentration of sperm at the baseline and week 16. One participant improved from a baseline of a Moderate concentration to Optimal at the end of the study. Two participants had an Optimal concentration in the beginning, which worsened to a Moderate reading at week 16.

Total sperm counts were obtained from semen volume and sperm concentration (Table 4). Three participants had increased counts of 106, 75, and 62 (in millions). The remaining two had decreases of 48 and 49 (in millions). Total sperm counts varied throughout the study with no consistent pattern.

\section{SHIM questionnaire}

Table 5: Changes in sexual health inventory for men (SHIM) $(n=6)$.

\begin{tabular}{|c|c|c|}
\hline & Mean \pm S.D. & $\begin{array}{c}\text { Number of subjects with abnormal } \\
\text { values ( } \mathbf{E 2 1 )}\end{array}$ \\
\hline Baseline & $19 \pm 1$ & 6 \\
\hline Week 4 & $17 \pm 6$ & 5 \\
\hline Week 8 & $17 \pm 7$ & 4 \\
\hline Week 12 & $15 \pm 9$ & 5 \\
\hline Week 16 & $21 \pm 2$ & 3 \\
\hline
\end{tabular}

Upon entry into the study, all participants had suboptimal SHIM scores (i.e., $\leq 21$ ); at the end, three of the six normalized (Table 5). During the study, mean SHIM scores started at $19 \pm 1$, trended downward, but increased to $21 \pm 2$ at week 16 . No one participant skewed the data and there was no relationship between SHIM scores and weight change. However, the only participant to gain weight also had a worse SHIM score at the end compared to baseline.

\section{General quality of life questionnaire}

Each week, the subjects rated nine questions about general quality of life issues, using a scale of one to five with five being the most favorable. Only summary data at baseline, week 4 , week 8 , week 12, and week 16 are presented (Table 6). The scores tended to be better for each quality of life indicator over the course of the study, except GI, which worsened over time for all subjects ( $4.3 \pm 0.5$ at baseline to $3.2 \pm 1.0$ at week $16 ; 26 \%$ worse). The greatest improvements of at least $20 \%$ between baseline and week 16 were seen in generally feeling better, energy level, diet quality, and passion.

Table 6: General quality of life questionnaire*.

\begin{tabular}{|c|c|c|c|c|c|}
\hline & Baseline & Week 4 & Week 8 & Week 12 & Week 16 \\
\hline $\begin{array}{c}\text { General } \\
\text { feeling }\end{array}$ & $2.8 \pm 0.8$ & $3.0 \pm 0.6$ & $3.3 \pm 1.0$ & $3.7 \pm 0.5$ & $\begin{array}{c}3.5 \pm 0.6 \\
(20 \%)\end{array}$ \\
\hline Feel full & $3.3 \pm 0.8$ & $3.5 \pm 0.6$ & $3.8 \pm 0.4$ & $3.7 \pm 0.5$ & $\begin{array}{c}3.5 \pm 0.6 \\
(6 \%)\end{array}$ \\
\hline Mood & $3.5 \pm 0.6$ & $3.5 \pm 0.6$ & $3.7 \pm 0.5$ & 4 & $4.0(12 \%)$ \\
\hline $\begin{array}{c}\text { Energy } \\
\text { level }\end{array}$ & $3.0 \pm 0.6$ & $3.3 \pm 0.5$ & $3.3 \pm 0.8$ & $3.7 \pm 0.5$ & $4.0(25 \%)$ \\
\hline $\begin{array}{c}\text { Gastroin- } \\
\text { testinal } \\
\text { disturbanc- } \\
\text { es }\end{array}$ & $4.3 \pm 0.5$ & $4.0 \pm 1.1$ & $3.7 \pm 0.8$ & $3.7 \pm 0.5$ & $\begin{array}{c}3.2 \pm 1.0 \\
(26 \%)\end{array}$ \\
\hline Sleep & 3 & $3.5 \pm 0.6$ & $3.5 \pm 0.6$ & $3.5 \pm 0.6$ & $\begin{array}{c}3.5 \pm 0.6 \\
(14 \%)\end{array}$ \\
\hline $\begin{array}{c}\text { Appear- } \\
\text { ance }\end{array}$ & $3.3 \pm 0.5$ & $3.7 \pm 0.5$ & $3.2 \pm 0.8$ & $3.7 \pm 0.5$ & $\begin{array}{c}3.7 \pm 0.5 \\
(11 \%)\end{array}$ \\
\hline Diet quality & $2.3 \pm 0.5$ & $3.8 \pm 0.8$ & $3.7 \pm 0.8$ & $3.8 \pm 0.4$ & $\begin{array}{c}3.4 \pm 0.8 \\
(12 \%)\end{array}$ \\
\hline Passion & $2.8 \pm 1.0$ & $3.7 \pm 1.0$ & $3.3 \pm 0.8$ & 4 & $\begin{array}{c}3.7 \pm 0.5 \\
(24 \%)\end{array}$ \\
\hline
\end{tabular}

${ }^{*}$ Responses were one to five, with five being the best. At week 16, the percentage change from baseline is presented. Of the percent change, only gastrointestinal disturbances worsened.

\section{Discussion}

Reduced libido and fertility are not only a condition of older men $[1,3]$. Younger men, with no underlying condition (medical or environmental like alcohol or illicit drug use) that can cause the problem, still experience sexual and reproductive health deficiencies. The results of this pilot study offer some hope for younger men who want to treat reduced libido with a dietary intervention. We found that the use of nutrient-dense, easyto-prepare functional foods helped overweight and obese men under age 50 years improve sexual performance in a variety of ways.

Both body weight and waist circumference reduced over 16 weeks. These changes resulted in a lower risk of chronic disease risk for hypertension, type 2 diabetes, and cardiovascular disease - all potential factors that can worsen libido $[2,18]$. We have demonstrated previously that use of nutrient-dense functional foods reduces body weight and waist circumference as well as mitigating disease risk $[13,14]$.

Subjective measures of sexual performance and quality of life improved. Although the SHIM results were on the cusp of still being sub-optimal at the end of the study, scores improved showing that the participants felt better about their sexual performance. 
It seems logical that testosterone would increase with weight loss. In this pilot study, both weight loss and increased salivary testosterone levels occurred. All participants experienced increased testosterone concentrations and by week 4, all had normal readings. Four of the six subjects lost weight. However, the effect of body weight and other anthropometric tests on total and calculated free testosterone levels has been challenged [19]. In a cross-sectional analysis of 821 men, testosterone and calculated free $\mathrm{T}$ were inversely correlated with fat mass, weight, body mass index, and waist/hip circumference. These findings were expected. But over a period of nearly five years, weight loss and loss of body fat did not affect baseline levels of these hormones.

The focus of the present study was not on having a successful conception, but semen volume and sperm concentrations were obtained. All but one participant had normal semen volumes throughout the study. Sperm concentrations varied throughout the study: three had higher counts; one remained the same; and one worsened. In addition, three had increased sperm concentrations and two experienced decreased values. These findings were likely due to the small sample size.

Beyond eating a healthy diet and maintaining a healthy body weight, little has been published on specific dietary guidelines to help overcome reduced libido. In contrast, more information is available on what diet to adopt by couples with impaired fecundity [20]. Adherence to healthy diets favoring seafood, poultry, whole grains, fruits, and vegetables are related to better fertility in women and better semen quality in men. Although these are sound recommendations, few people adhere to such a healthy diet [21]. Americans consume inadequate amounts of all these foods according to the Dietary Guidelines for Americans 2015-2020. The easy-to-prepare nutrient-dense functional foods used in this study contain the same nutrients (e.g., vitamins, minerals, and omega- 3 fatty acids) as the foods recommended to overcome impaired fecundity. The men in this study consumed four to five functional foods daily over four months and found them easy to incorporate into their lives. It is possible that the nutrient-dense functional foods used in this study could also help couples with difficulty conceiving alongside the observed improvement in libido. These functional foods promote weight loss as observed in this present study and elsewhere, which is related to reproductive health [6-9, 13-14].

The study limitations are its small sample size, but we are accruing more participants now. Also, the study was not randomized but enough objective data were obtained serially (i.e., baseline and monthly for testosterone, semen volume, and sperm concentration) to compensate for this. Home testing of salivary testosterone, semen volume, and sperm concentration were used but this was not considered to be a limitation. The test kits used in this study have been validated against standard methods and are within the limits of acceptable testing when compared to blood for testosterone, and semen and sperm concentrations obtained in a physician's office [16,17]. The companies offering these home-testing kits are certified by the Clinical Laboratory Improvement Amendments (CLIA) (https:// www.cms.gov/Regulations-and index.html).

\section{Conclusions}

In summary, this pilot study demonstrated that nutrientdense functional foods helped men with reduced libidos as far as producing weight loss, improvements in subjective measures of sexual performance and quality of life, testosterone levels, and to a lesser effect, better sperm concentrations. More subjects are needed to confirm these positive findings.

\section{Acknowledgements}

We are grateful to the men that participated in this study. It was long and they had to consume most of their diet from nutrient-dense functional foods provided. And, the study involved multiple tests of semen and salivary testosterone, which the group dutifully did with grace. A special thanks go to Rochelle Ramacher, one of our exceptional coaches, who helped the men adhere to the study protocol; and Andrea Franz, who oversaw technical support related to data collection.

\section{Conflicts of Interest}

Ms. Bauer, Mr. Marlsand, and Drs. Wijendran and Bell are full-time employees of Nutrient, the company that made the nutrient-dense foods used in this study. Dr. Sommer is the Chief Science Officer of Sandstone Diagnostics, Inc., the company that manufactures the semen volume and sperm concentration measuring device used in this study. Dr. Francis, at the time that this study was conducted, was the Executive Medical Director for EverlyWell, the company that manufactures the salivary testosterone testing kit used in this study.

\section{Declaration of Financial Support}

Nutrient Foods, LLC, Reno, Nevada funded the study (http://nutrientfoods.com) by providing the functional foods and purchasing the testosterone and semen testing kits. Both Sandstone Diagnostics, Inc. and Everly Well provided their home testing kits at a reduced rate to Nutrient.

\section{References}

1. Araujo AB, Esche GR, Kupelian V, O'Donnell AB, Travison TG, et al. (2007) Prevalence of Symptomatic Androgen Deficiency in Men. J Clin Endocrinol Metab 92(11): 4241-4247.

2. Wijesinha S, Piterman L, Kirby C (2013) The male reproductive system. A overview of common problems. Aust Fam Physician 42(5): 276-278

3. Travison TG, Araujo AB, O’Donnell AB, Kupelian V, McKinlay JB, et al. (2007) A Population-Level Decline in Serum Testosterone Levels in American Men. J Clin Endocrinol Metab 92(1): 196-202.

4. Millar AC, Lau ANC, Tomlinson G, Kraguljac A, Simel DL, et al. (2016) Predicting low testosterone in aging men: a systematic review. CMAJ 188(13): E321-E330. 


\section{Current Research in Diabetes \& Obesity Journal}

5. Levine H, Jorgensen N, Martino-Andrade A, Mendiola J, Weksler-Derri D, et al. (2017) Temporal trends in sperm count: a systematic review and meta-regression analysis. Hum Reprod Update 23(6): 646-659.

6. Liu Y, Ding Z (2017) Obesity, a serious etiologic factor for male subfertility in modern society. Reproduction 154(4): R123-R131.

7. Sermondade N, Faure C, Fezeu L, Shayeb AG, Bonde JP, et al. (2013) BMI in relation to sperm count: an updated systematic review and collaborative meta-analysis. Hum Reprod Update 19(3): 221-231.

8. Eisenberg ML, Kim S, Chen Z, Sundaram R, Schisterman EF, et al. (2014) The relationship between male BMI and waist circumference on semen quality: data from the LIFE study. Hum Reprod 29(2): 193-200.

9. Ramaraju GA, Teppala S, Prathigudupu K, Kalagara M, Thota S, et al (2018) Association between obesity and sperm quality. Andrologia 50(3): e12888.

10. Erectile dysfunction: patient guide. The American Urological Association

11. Durairajanayagam D (2018) Lifestyle causes of male infertility. Arab J Urol 16(1): 10-20.

12. Therapeutic Products Programme and the Food Directorate from the Health Protection Branch. ARCHIVED - Policy Paper - Nutraceuticals/ Functional Foods and Health Claims on Foods.

13. Bell SJ, Ling PR, Marsland C (2016) Nutrient-Dense, Portion-Controlled Meals and Snacks Promote Weight Loss. J Food Technol Nutr Sci 2(1).

This work is licensed under Creative Commons Attribution 4.0 Licens DOI: 10.19080/CRDOJ.2019.12.555841
14. Bell SJ, Ling PR, Baker RA, Marsland C (2018) Use of self-reported data to determine the effect of nutrient-dense meals on body weight and quality of life. Journal of Nutrition \& Food Sciences 8: 3.

15. Cappelleri JC, Rosen RC (2005) The Sexual Health Inventory for Men (SHIM): a 5-year review of research and clinical experience. Int J Impot Res 17: 307-319.

16. Keevill BG, MacDonald P, Macdowall W, Lee DM, Wu FC, et al. (2014) Salivary Testosterone Measurement by Liquid Chromatography Tandem Mass Spectrometry in Adult Males and Females. Ann Clin Biochem 51(Pt 3): 368-378.

17. Schaff UY, Fredriksen LL, Epperson JG, Quebral TR, Naab S, et al. (2017) Novel centrifugal technology for measuring sperm concentration in the home. Fertil Steril 107(2): 358-364.

18. National Heart, Lung, and Blood Institute. Classification of Overweight and Obesity by BMI, Waist Circumference, and Associated Disease Risks.

19. Gates MA, Mekary RA, Chiu GR, Ding EL, Wittert GA, et al. (2013) Sex Steroid Hormone Levels and Body Composition in Men. J Clin Endocrinol Metab 98(6): 2442-2450.

20. Gaskins AJ, Chavarro JE (2018) Diet and fertility: a review. Am J Obstet Gyneco 218(4): 379-389.

21. Dietary Guidelines for Americans 2015-2020. $8^{\text {th }}$ (edn).

Your next submission with Juniper Publishers will reach you the below assets

- Quality Editorial service

- Swift Peer Review

- Reprints availability

- E-prints Service

- Manuscript Podcast for convenient understanding

- Global attainment for your research

- Manuscript accessibility in different formats

( Pdf, E-pub, Full Text, Audio)

- Unceasing customer service

Track the below URL for one-step submission

https://juniperpublishers.com/online-submission.php 